\title{
The spinal cord independence measure (SCIM): Sensitivity to functional changes in subgroups of spinal cord lesion patients
}

\author{
A Catz ${ }^{* 1}$, M Itzkovich ${ }^{1}$, E Agranov ${ }^{1}, \mathrm{H} \mathrm{Ring}^{1}$ and A Tamir ${ }^{2}$ \\ ${ }^{1}$ Loewenstein Rehabilitation Hospital, Raanana and Sackler Faculty of Medicine, Tel Aviv University, Tel Aviv, \\ Israel; ${ }^{2}$ Department of Community Medicine, Faculty of Medicine, Technion, Haifa, Israel
}

\begin{abstract}
Background: The spinal cord independence measure (SCIM) is a newly developed disability scale specific to patients with spinal cord lesions (SCL). Its sensitivity to functional changes in a whole cohort of SCL patients was found to be better than that of the functional independence measure (FIM).

Objective: To compare the sensitivity to functional changes of the SCIM and the FIM in SCL subgroups.

Design: A comparative self-controlled study.

Setting: The Spinal Department, Loewenstein Rehabilitation Hospital, Raanana, Israel.

Subjects: 22 SCL inpatients.

Interventions: Monthly SCIM and FIM assessments of the subgroups.

Main outcome measures: Functional change detection rate (FDR) and mean differences between consecutive scores (DCS).

Results: The outcome measures of the SCIM were higher than those of the FIM for tetraplegia and paraplegia, complete and incomplete lesions (the FIM missed $25-27 \%$ of the functional changes detected by the SCIM; DSC 8.2-11.4 vs 5.2-9; $P<0.05$ in most comparisons). The SCIM did not exhibit this advantage, however, in the functional areas of self-care and mobility in the room and toilet. Further subgrouping yielded similar results.

Conclusions: The SCIM is more sensitive than the FIM to functional changes in the subgroups studied, and has the potential to serve as a universal tool for disability assessment of SCL patients.
\end{abstract}

Spinal Cord (2001) 39, 97-100

Keywords: SCIM; subgroups; spinal cord; disability assessment

\section{Introduction}

The spinal cord independence measure (SCIM), an innovative disability scale, was developed in the Spinal Department of Loewenstein Rehabilitation Hospital. The SCIM is a rating instrument for measuring the meaningful functional changes in patients with spinal cord lesions (SCL). ${ }^{1}$ Other currently used disability scales, such as the modified Barthel index $(\mathrm{MBI})^{2}$ and the functional independence measure (FIM), ${ }^{3}$ were formulated for various disorders, and are not sensitive enough to assess the specific functional problems of SCL patients. ${ }^{1,4,5}$

The SCIM covers three areas of function: self-care (score range $0-20$ ), respiration and sphincter management $(0-40)$, and mobility $(0-40)$. Mobility was scored in the room and toilet and indoors and

*Correspondence: A Catz, Department IV, Spinal Rehailitation, Loewenstein Rehabilitation Hospital, 278 Achuza St., P.O. Box 3, Raanana 43100, Israel outdoors. The final score ranges between 0 and $100{ }^{1}$ In an earlier work, the SCIM exhibited a high interrater reliability and better sensitivity than the FIM to functional changes in SCL patients. ${ }^{1}$ However, these findings were based on analysis of the data for the entire study population, and further analysis was needed to determine the scale's usefulness in specific subgroups.

For this purpose, SCIM and FIM scores were studied in patients with tetraplegia, paraplegia, complete lesions, and incomplete lesions. We hypothesized that the SCIM is more sensitive than the FIM to functional changes in all subgroups.

\section{Methods}

The study population consisted of 22 SCL inpatients (17 males, five females) in the Spinal Department of Loewenstein Rehabilitation Hospital, for whom complete data were available. Eight had tetraplegia and 14 
paraplegia; six had complete or almost complete lesions (Frankel A or B) and 16 had incomplete lesions (Frankel $\mathrm{C}$ or D). Ages ranged between 23 and 76 years $($ mean $=48.1, \mathrm{SD}=16.2)$. Eleven of the spinal lesions were traumatic, and the remainder were compressive degenerative spinal lesions, meningioma, or achondroplasia.

All patients were evaluated with the SCIM and the FIM for the first time 1 week after admission to the department and thereafter every month during hospitalization. Follow-up ranged from 1 to 6 months (average $=3$ months). Each area of function on the SCIM was scored by a pair of staff members in the relevant field: Self-care: occupational therapists; respiration and sphincter management and mobility in the room and toilet: nurses; mobility indoors and outdoors: physical therapists. Each member of the scoring pair was blinded to the other's results. Only assessment batteries which were completed within 1 week of each other, were included in the analysis.

To determine the sensitivity of the scale to functional changes within the subgroups, the SCIM functional fluctuations measured by the staff were compared to the FIM functional fluctuations measured by a nurse throughout the duration of rehabilitation. We compared both the rate of detection of functional changes (FDR) (the number of changes in scoring divided by the number of comparisons between consecutive test batteries) and the mean difference between consecutive scores (DCS).

Scores range for the SCIM is $1-100$, and for the FIM 18-126. We therefore normalized the FIM score $F$ to $F^{*}$, using the formula: $F^{*}=(F-18) /(126-18) \times 100$. Data were analyzed with the SPSS, using the McNemar and Wilcoxon Signed Ranks tests. ${ }^{6}$

\section{Results}

All functional fluctuations detected by the FIM total score were also detected by the SCIM total score in the subgroups with tetraplegia, paraplegia, complete lesions and incomplete lesions. However, FIM missed $25-27 \%$ of the functional changes detected by the SCIM (Table 1). The difference in FDR reached statistical significance in the subgroups of tetraplegia, paraplegia, and incomplete lesions $(P<0.02)$. Comparison of the total scores in consecutive evaluations yielded significantly higher mean differences for the SCIM in all the mentioned subgroups (DCS $=8.2-11.4$ vs $5.2-9.0 ; P<0.05$ ) (Table 2).

When the change in individual areas of function was examined, the FDR and the DCS were significantly higher for the SCIM than for the FIM, for respiration and sphincter management

Table 1 The SCIM-FIM difference in the functional change detection rate (FDR) in SCL subgroups

\begin{tabular}{lcccc}
\hline & Tetraplegia & Paraplegia & Complete lesions & Incomplete lesions \\
\hline N & 22 & 35 & 16 & 41 \\
N SCIM-A $(\%)(P)$ & $6(27)(0.02)$ & $9(26)(0.005)$ & $4(25)(\mathrm{NS})$ & $11(27)(0.005)$ \\
N SCIM-S $(\%)(P)$ & $3(14)(\mathrm{NS})$ & $6(17)(\mathrm{NS})$ & $3(19)(\mathrm{NS})$ & $6(15)(\mathrm{NS})$ \\
N SCIM-R $(\%)(P)$ & $9(41)(0.02)$ & $23(66)(0.0001)$ & $13(81)(0.0001)$ & $19(46)(0.0001)$ \\
N SCIM-I $(\%)(P)$ & $7(32)(0.05)$ & $10(29)(0.05)$ & $4(25)(\mathrm{NS})$ & $13(32)(0.005)$ \\
N SCIM-O $(\%)(P)$ & $10(46)(0.01)$ & $16(46)(0.0001)$ & $5(31)(0.05)$ & $21(51)(0.0001)$ \\
\hline
\end{tabular}

SCL: spinal cord lesion; N: number of scorings compared; N SCIM: number of functional changes detected by SCIM only (A: total score; S: self-care; R: respiration and sphincter management; I: mobility in room and toilet; O: mobility indoors and outdoors); $P$ : $P$ value in the McNemar test, NS=non-significant

Table 2 Mean differences between consecutive scores (DCS) of FIM and SCIM

\begin{tabular}{|c|c|c|c|c|}
\hline & Tetraplegia & Paraplegia & Complete lesions & Incomplete lesions \\
\hline $\mathrm{N}$ & 22 & 35 & 16 & 41 \\
\hline Mean $\mathrm{F}^{*} \mathrm{DCS}(\mathrm{SD})$ & $5.2(12.1)$ & $9.0(10.2)$ & $5.9(5.1)$ & $8.2(12.6)$ \\
\hline Mean SCIM DCS (SD) & $8.2(13.3)$ & $11.4(9.2)$ & $10.1(8.9)$ & $10.2(11.8)$ \\
\hline$\Delta \mathrm{SF}-\mathrm{A}(P)$ & $3.0(0.02)$ & $2.4(0.05)$ & $4.2(0.02)$ & $2.0(0.05)$ \\
\hline$\Delta \mathrm{SF}-\mathrm{S}(P)$ & $-0.9(\mathrm{NS})$ & $-2.9(\mathrm{NS})$ & $-1.6(\mathrm{NS})$ & $-2.3(\mathrm{NS})$ \\
\hline$\Delta \mathrm{SF}-\mathrm{R}(P)$ & $1.6(0.01)$ & $3.6(0.0001)$ & $4.4(0.002)$ & $2.2(0.0001)$ \\
\hline$\Delta$ SF-I $(P)$ & $-0.5(\mathrm{NS})$ & $1.4(\mathrm{NS})$ & $-0.7(\mathrm{NS})$ & $-1.2(\mathrm{NS})$ \\
\hline$\Delta \mathrm{SF}-\mathrm{O}(P)$ & $2.4(0.03)$ & $2.3(0.0005)$ & $1.4(0.02)$ & $2.7(0.0005)$ \\
\hline
\end{tabular}

$\mathrm{F}^{*}$ : Fim normalized score; N: number of scorings compared; $\triangle \mathrm{SF}$ : the difference between mean SCIM DCS and mean FIM DCS in each subgroup and area of function (A: total score; S: self-care; R: respiration and sphincter management; I: mobility in room and toilet; $\mathrm{O}$ : mobility indoors and outdoors); $P$ : $P$ value in the Wilcoxon Signed Ranks test; NS: non-significant 
and mobility indoors and outdoors, in all these subgroups $(P<0.05)$. Examination of changes in the other two areas of function in these subgroups could not demonstrate significantly higher SCIM functional change measures (Tables 1, 2). Further subgrouping into complete tetraplegia, incomplete tetraplegia, complete paraplegia and incomplete paraplegia, yielded similar results for the last three subgroups: changes in both FDR and DCS were usually significantly higher for the SCIM than for the FIM $(P<0.05)$, except for the functional areas of self-care and mobility in the room and toilet (Tables 3, 4). The group of complete tetraplegia could not be included in the analysis, as it consisted of only one patient.

\section{Discussion}

The division of the SCL population into subgroups reduced the number of items available for each comparison, with a consequent decrease in the chance of displaying statistical significance for differences between the SCIM and the FIM. Nevertheless, the absolute values of the total scores FDR and the mean DCS were higher for the SCIM than for the FIM in all the examined subgroups, and the differences between scales reached significance on most of the statistical tests. The results, therefore, support the study hypothesis that the SCIM is more sensitive than the FIM to functional changes in subgroups of SCL patients. This finding, combined with those of a previous study that demonstrated the SCIM's high interrater reliability and sensitivity to functional changes, supports the validity of the new scale. ${ }^{1}$

When tested in individual areas of function, the advantage of the SCIM over the FIM was similar in subgroups to that found in a whole cohort. ${ }^{1}$ The SCIM was more sensitive to functional changes in respiration and sphincter management and in mobility indoors and outdoors, but not in self-care and mobility in the room and toilet. Changes in the formulation of the last two SCIM areas, are included in the second version of the scale, which is currently being investigated.

Additional subdivision of the present study population left very small groups and further limited the possibility of statistical analysis. However, the comparisons of FDR and DCS in the subgroups of incomplete tetraplegia, incomplete paraplegia, and complete paraplegia, support the conclusion that the SCIM may be applicable to various SCL patients. The authors believe that further refining of the scale will prove the SCIM to be a universal tool for assessing the everyday performance of this population.

Table 3 The SCIM-FIM difference in the functional change detection rate (FDR) in further SCL subgrouping

\begin{tabular}{lccc}
\hline & Tetraplegia incomplete & Paraplegia incomplete & Paraplegia complete \\
\hline N & 21 & 20 & 15 \\
N SCIM-A $(\%)(P)$ & $5(24)(0.04)$ & $6(30)(0.03)$ & $3(20)(\mathrm{NS})$ \\
N SCIM-S $(\%)(P)$ & $3(14)(\mathrm{NS})$ & $3(15)(\mathrm{NS})$ & $3(20)(\mathrm{NS})$ \\
N SCIM-R $(\%)(P)$ & $8(38)(0.02)$ & $11(55)(0.005)$ & $12(80)(0.0001)$ \\
N SCIM-I $(\%)(P)$ & $7(33)(0.04)$ & $6(30)(\mathrm{NS})$ & $4(27)(\mathrm{NS})$ \\
N SCIM-O $(\%)(P)$ & $10(48)(0.01)$ & $11(55)(0.0005)$ & $5(33)(0.04)$ \\
\hline
\end{tabular}

SCL: spinal cord lesion; N: number of scorings compared; N SCIM: number of functional changes detected by SCIM only (A: total score; S: self-care; R: respiration and sphincter management; I: mobility in room and toilet; O: mobility indoors and outdoors); $P$ : $P$ value in the McNemar test; NS: non-significant

Table 4 Mean differences between consecutive scores (DCS) of FIM and SCIM in further SCL subgrouping

\begin{tabular}{lccc}
\hline & Tetraplegia incomplete & Paraplegia incomplete & Paraplegia complete \\
\hline N & 21 & 20 & 15 \\
Mean F* DCS (SD) & $5.5(12.3)$ & $11.1(12.5)$ & $6.3(5.0)$ \\
Mean SCIM DCS (SD) & $8.4(13.6)$ & $12.1(9.4)$ & $10.4(9.1)$ \\
$\Delta$ SF-A $(P)$ & $2.9(0.03)$ & $1.0(\mathrm{NS})$ & $4.1(0.02)$ \\
$\Delta$ SF-S $(P)$ & $-1.0(\mathrm{NS})$ & $-3.8(\mathrm{NS})$ & $-1.7(\mathrm{NS})$ \\
$\Delta$ SF-R $(P)$ & $1.4(0.01)$ & $3.0(0.0002)$ & $4.4(0.003)$ \\
$\Delta$ SF-I $(P)$ & $-0.6(\mathrm{NS})$ & $-1.9(\mathrm{NS})$ & $-0.8(\mathrm{NS})$ \\
$\Delta$ SF-O $(P)$ & $2.5(0.03)$ & $2.9(0.0005)$ & $1.5(0.02)$ \\
\hline
\end{tabular}

$\mathrm{F}^{*}$ : Fim normalized score; N: number of scorings compared; $\triangle \mathrm{SF}$ : the difference between mean SCIM DCS and mean FIM DCS in each subgroup and area of function (A: total score; S: self-care; R: respiration and sphincter management; I: mobility in room and toilet; $\mathrm{O}$ : mobility indoors and outdoors); $P$ : $P$ value in the Wilcoxon Signed Ranks test; NS: non-significant 


\section{Acknowledgements}

This study was supported by the Unit of Medical Services, Rehabilitation Department, Ministry of Defence (Grant 1-1006-94).

\section{References}

1 Catz A et al. SCIM - spinal cord independence measure: a new disability scale for patients with spinal cord lesions. Spinal Cord 1997; 35: 850-856.

2 McDowell I, Newell C. Measuring Health: A Guide to Rating Scales and Questionnaires. Oxford University Press: Oxford 1987, pp $49-54$.
3 Uniform Data Set for Medical Rehabilitation, Version 3.0. Data Management Service of the Uniform Data System for the Medical Rehabilitation and the Center for Functional Assessment Research. SUNY: Buffalo, New York 1990.

4 Gresham GE et al. The quadriplegia index of function (QIF): sensitivity and reliability demonstrated in a study of thirty quadriplegic patients. Paraplegia 1986; 24: $38-44$.

5 Marino RJ et al. Assessing self care status in quadriplegia: comparison of the quadriplegia index of function (QIF) and the functional independence measure (FIM). Paraplegia 1993; 31: $225-233$.

6 Siegel S, Castellan NH. Nonparametric Statistics for The Behavioral Sciences. McGraw-Hill: New York 1988. 